\title{
IPSS in cyclical cushing's syndrome due to ectopic ACTH production: proof of principle and potential diagnostic pitfall
}

\begin{abstract}
Introduction: Cyclical Cushing's syndrome (CCS) has been described in patients with excessive endogenous cortisol produced in fluctuating rhythmical patterns. Although most cases of CCS have been reported secondary to an adrenocorticotropic hormone (ACTH) producing pituitary adenoma, there have also been reports of CCS caused by ectopic ACTH producing tumors. Due to its fluctuating nature, the clinical and biochemical diagnosis of CCS represents a challenge.

Case presentation: A 53year-old man undergoing evaluation for ACTH-dependent CCS underwent inferior petrosal sinus sampling (IPSS). The peak inferior petrosal to peripheral ACTH gradient level was consistent with a false-positive diagnosis for Cushing's disease (CD). However, results showed suppression of the hypothalamic-pituitary-adrenal (HPA) axis from antecedent hypercortisolism, therefore the IPSS was deemed non-diagnostic. Repeat IPSS during another cycle of hypercortisolism a few months later, confirmed the diagnosis of ectopic ACTH production. The source was localized to the lung and the patient underwent resection of a typical bronchial carcinoid tumor staining for ACTH. The patient achieved complete biochemical and clinical resolution of Cushing's syndrome (CS).

Discussion and conclusions: While the potential for IPSS to result in diagnostic misclassification of the etiology of CCS has been brought up in the literature, to the best of our knowledge, this is the first report of IPSS performed both in and out of cycle in a patient with CCS due to ectopic ACTH syndrome. The findings could provide "proof-of-principle" as to the expected findings and the potential for false-positive results leading to erroneous diagnosis of pituitary etiology of CCS.
\end{abstract}

Keywords: cyclical cushing's syndrome, cushing's syndrome, inferior petrosal sinus sampling (IPSS), diagnosis, ectopic ACTH.
Volume 5 Issue 4 - 2017

\author{
Zeina C Hannoush,' Juan D Palacios,' Dileep \\ Yavaga',2 Anup K Sabharwal,' AtilY Kargi' \\ 'Department of Medicine, University of Miami Miller School of \\ Medicine, USA \\ ${ }^{2}$ Department of Neurology and Neurosurgery, University of \\ Miami Miller School of Medicine, USA
}

Correspondence: AtilY Kargi, Division of Endocrinology, Diabetes and Metabolism, Department of Medicine, University of Miami Miller School of Medicine, Miami, FL 33|36, USA, Emailakargi@med.miami.edu

Received: July 23, 2017 | Published: October 4, 2017
Abbreviations: CCS, cyclical cushing's syndrome; CD, cushing's disease; ACTH,

adrenocorticotropic hormone; IPSS, inferior petrosal sinus sampling; HPA, hypothalamic-pituitary-adrenal; CRH, corticotropinreleasing hormone.

\section{Introduction}

Cyclical Cushing's syndrome (CCS) has been described in patients with Cushing's syndrome in which excessive endogenous cortisol is produced in fluctuating rhythmical patterns. ${ }^{1}$ \{Mullan, $2007 \# 16$. This syndrome can be associated with fluctuating signs and symptoms; cycle lengths have been described between 12 hours and 85 days. ${ }^{2}$ CCS was initially believed to be very rare, but recent evidence has shown that it might occur more frequently that predicted. ${ }^{3}$ Although most cases of CCS have been reported secondary to an adrenocorticotropic hormone (ACTH) producing pituitary ad+enoma, there have also been reports of adrenal adenomas, ectopic typical and atypical carcinoids, pheochromocytomas and other ectopic ACTH producing tumors. ${ }^{2}$ The pathophysiologic mechanisms behind the fluctuations in hormone production are not well understood although different mechanisms have been proposed based on findings from case reports. ${ }^{1}$

The clinical and biochemical diagnosis of CCS can represent a challenge for the clinician. Here we report the case of a 53yearold man with cyclical Cushing's syndrome due to an ectopic ACTH producing typical bronchial carcinoid tumor and discuss the challenges in its diagnosis, focusing on the potential diagnostic pitfall in the interpretation of the inferior petrosal sinus sampling (IPSS) which if performed "out-of-cycle" can lead to misleading conclusions regarding the source of excess ACTH production.

\section{Case presentation}

A 53year-old man with history of recent onset hypertension and type 2 diabetes mellitus, presented with progressively worsening symptoms of depression, lack of energy, muscle weakness and hypokalemia. He was admitted to an outside hospital with altered mental status due to severe hypokalemia and hyperammonemia with normal liver function. Laboratory workup revealed significant hypercortisolism: $24 \mathrm{~h}$ urine free cortisol (UFC) $>4000 \mathrm{mcg} / 24 \mathrm{~h}$ $(\mathrm{n}<55 \mathrm{mcg} / 24 \mathrm{~h})$, AM cortisol of $60 \mathrm{mcg} / \mathrm{dL}$ (n $4-22 \mathrm{mcg} / \mathrm{dL})$, PM cortisol of $30 \mathrm{mcg} / \mathrm{dL}$, elevated ACTH level of $257 \mathrm{pg} / \mathrm{mL}$ (n $6-50 \mathrm{pg} /$ $\mathrm{mL}$ ) with failure to suppress cortisol after low and high dose dexamethasone suppression testing, normal DHEA-S and thyroid function tests. Whole body CT scan and octreotide scan as well as pituitary MRI failed to reveal any tumors, the adrenal glands where found to have bilateral hypertrophy without discrete lesions. The patient had no history of receiving exogenous steroids, denied use of valproic acid, ethanol, or other substances. After his discharge the patient improved significantly going back to his clinical baseline. At that time in the outpatient setting he had normal laboratory values with a $24 \mathrm{~h}$ UFC of $21 \mathrm{mcg} / 24 \mathrm{~h}$, AM serum cortisol of $17.4 \mathrm{mcg} / \mathrm{dL}$, ACTH of $46 \mathrm{pg} / \mathrm{mL}$, DHEA $104 \mathrm{ug} / \mathrm{dL}$, aldosterone of $10 \mathrm{ng} / \mathrm{dL}$ and plasma renin activity of $3.27 \mathrm{ng} / \mathrm{mL} / \mathrm{hr}$ while on a beta blocker and normal electrolytes. Pertinent findings on physical exam included 
no moon facies but slight plethora, some supraclavicular fullness, no dorsocervical fat pad, obese abdomen with no organomegaly and no striae.

Follow up laboratory workup revealed significantly elevated levels of cortisol and ACTH alternating with normal values. The patient was observed to have symptoms of irritability, mental cloudiness, plethora and worsening hypertension during the episodes of hypercortisolism. Cycles of hypercortisolism would occur approximately every 4 months and last almost 4 weeks. The patient was scheduled for IPSS. 2 weeks prior to IPSS: UFC $>2400 \mathrm{mcg} / 24 \mathrm{~h}$, plasma ACTH concentration of $190 \mathrm{pg} / \mathrm{mL}$ and 8 A.M. serum cortisol $35.9 \mathrm{mcg} / \mathrm{dL}$. Hypercortisolemia was re-confirmed 5 days prior to IPSS.

During IPSS baseline ACTH concentrations were similar at femoral vein $(14 \mathrm{pg} / \mathrm{mL})$, right IPS $(11 \mathrm{pg} / \mathrm{mL})$ and left IPS $(17 \mathrm{pg} /$ $\mathrm{mL}$ ) (Table 1). Following corticotropin-releasing hormone (CRH) stimulation, ACTH levels peaked at $41 \mathrm{pg} / \mathrm{mL}$ in the right IPS and $30 \mathrm{pg} / \mathrm{mL}$ in the left IPS while there was no increase in ACTH at the femoral vein. The peak IPS:P ratios were 4.1 on the right and 2.4 on the left. Cortisol levels remained low $(<4.2 \mathrm{mcg} / \mathrm{dL})$ after CRH stimulation. Though peak IPS:P ACTH gradient was $>3$, commonly accepted as indicative of Cushing's disease (CD), the results were interpreted as consistent with suppression of the HPA axis from antecedent hypercortisolism, therefore the IPSS was deemed as nondiagnostic for subtype classification of ACTH-dependent CS.

Table I Results of first IPSS performed "out-of-cycle" with low ACTH and cortisol measurements at all sites sampled and all time points with increase of ACTH in the petrosal sinuses after $\mathrm{CRH}$ stimulation yielding a peak IPS: P ACTH gradient $>4$. Units for serum concentrations: ACTHng/mL, Cortisolmcg/dL

\begin{tabular}{|c|c|c|c|c|c|c|}
\hline \multirow{2}{*}{$\begin{array}{l}\text { Site } \\
\text { Minutes } \\
\text { after } \\
\text { CRH }\end{array}$} & \multicolumn{2}{|c|}{ Femoral vein } & \multirow{2}{*}{$\begin{array}{l}\text { Right } \\
\text { IPS } \\
\text { ACTH }\end{array}$} & \multirow{2}{*}{$\begin{array}{l}\text { Left } \\
\text { IPS } \\
\text { ACTH }\end{array}$} & \multirow{2}{*}{$\begin{array}{l}\text { Right } \\
\text { IPS:P } \\
\text { ACTH }\end{array}$} & \multirow{2}{*}{$\begin{array}{l}\text { Left } \\
\text { IPS:P } \\
\text { АСТH }\end{array}$} \\
\hline & АСТH & Cortisol & & & & \\
\hline $0^{\prime}$ & 14 & & 11 & 17 & 0.8 & 1.2 \\
\hline 2' & 8 & 3.3 & 13 & 10 & 1.6 & 1.3 \\
\hline 5 , & 11 & & 12 & & 1.1 & \\
\hline $10^{\circ}$ & 11 & 3.6 & 36 & 14 & 3.3 & 1.3 \\
\hline 15' & 10 & 3.5 & 41 & 24 & 4.1 & 2.4 \\
\hline 30 ' & 13 & 4.1 & 19 & 30 & 1.5 & 2.3 \\
\hline
\end{tabular}

The patient presented with another cycle of severe hypercortisolism over 4months after the initial IPSS. Repeat IPSS showed elevated baseline ACTH $(99 \mathrm{pg} / \mathrm{mL})$ and cortisol $(45 \mathrm{mcg} / \mathrm{dL})$ levels at the femoral vein (Table 2). Due to thrombotic occlusion, the left IPS was unable to be accessed during the second IPSS procedure. All IPS:P ratios were $<1.4$ before and after $\mathrm{CRH}$ administration and $\mathrm{ACTH}$ and cortisol levels did not rise at any site.

The patient was diagnosed as ectopic ACTH syndrome (EAS). Further imaging with repeat octreoscan and FDG-PET/CT were negative, the patient was subsequently referred to National Institutes of Health in Bethesda, Maryland for further imaging investigation which included lung MRI, F-DOPA PET and Gallium dotatate PET imaging studies which revealed a $1 \mathrm{~cm}$ right middle lobe pulmonary lesion and the patient underwent right middle lobe wedge lung nodule lobectomy revealing a $1.3 \mathrm{~cm}$ typical bronchial carcinoid tumor staining for ACTH (Figure 1). Postoperatively, he had low cortisol levels and was started on physiologic replacement dose of hydrocortisone that in a period of 3 months was safely tapered until discontinued. The patient showed complete resolution of his signs and symptoms of Cushing's syndrome and continues to be on biochemical remission after 2years.

Table 2 Results of second IPSS performed "in cycle" demonstrating elevated ACTH (reference range 6-55pg/mL) and hypercortisolemia. No significant IPS: PACTH gradient was observed before or after $\mathrm{CRH}$ administration and there was no significant increase in ACTH or cortisol following $\mathrm{CRH}$ stimulation. Results were consistent with an ectopic source of ACTH production. The left inferior petrosal sinus was not accessible due to occlusion from thrombus. Units for serum concentrations: ACTHng/mL, Cortisolmcg/dL

\begin{tabular}{lllll}
\hline Site & & Femoral Vein & Right IPS & Right IPS:P \\
\hline $\begin{array}{l}\text { Minutes } \\
\text { after CRH }\end{array}$ & ACTH & Cortisol & ACTH & \\
\hline $0^{\prime}$ & 99 & 44 & 80 & 0.8 \\
$2^{\prime}$ & 82 & 43 & & \\
$5^{\prime}$ & 79 & & 84 & 1.1 \\
$10^{\prime}$ & 69 & 45 & 63 & 0.9 \\
$15^{\prime}$ & 70 & 44 & 51 & 0.7 \\
$30^{\prime}$ & 73 & 39 & 78 & 1.1 \\
\hline
\end{tabular}
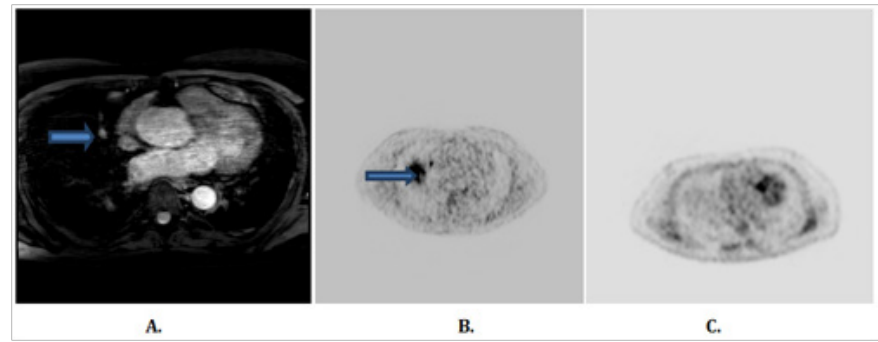

Figure I Results of imaging studies for the investigation of ectopic ACTH production. (A) Lung MRI demonstrates I $\mathrm{cm}$ right middle lobe lung nodule; (B) F-DOPA PET image demonstrating increased radiotracer uptake in the location consistent with the lung nodule on MRI; (C) FDG-PET scan does not show a focus of increased activity in the lungs.

\section{Discussion and conclusion}

Cyclical Cushing's syndrome often poses a diagnostic challenge. It should be suspected in patients with typical features of Cushing's syndrome with normal or variable biochemical findings of ACTH and cortisol level, in cases of clear signs and symptoms of CS and evidence of adrenal insufficiency and patients with biochemical evidence of hypercortisolism without overwhelming clinical features of CS. Exogenous cortisol intake, pseudo-Cushing's states, glucocorticoid resistance and macronodular adrenal hyperplasia should be ruled out in these situations as part of the differential diagnosis. ${ }^{4}$

CCS has been defined as a biochemical finding of at least three peaks and two troughs of cortisol production, ${ }^{5}$ but variabilities in the inter-cyclic period brings a substantial limitation to the diagnosis. Not all patients with CCS have consistent cycle length and we would propose a change in nomenclature for such cases from CCS to the more accurate term "episodic hypercortisolism". High-dose dexamethasone suppression test can be misleading and there have been cases reported with paradoxical response. ${ }^{2}$ Frequent repeat collection of $24 \mathrm{~h}$ urine for free cortisol measurement is inconvenient for many patients. Different diagnostic approaches have been proposed including measurement of cortisol to creatinine ratio of morning urine samples 
for 28 consecutive days, late night salivary cortisol measurement during the same time period, ${ }^{6}$ as well as the use of the desmopressin test, ${ }^{4}$ and new procedures that have now become available such as the measurement of hair cortisol levels. ${ }^{7}$ However multiple concerns remain about the precision and accurate interpretation of these tests.

Once the diagnosis of ACTH dependent Cushing's syndrome has been established, bilateral inferior petrosal sinus sampling can be performed to try differentiating central (pituitary) from ectopic sources of ACTH production. ${ }^{8}$ However, this procedure can be unreliable in CCS unless performed when the syndrome is active. It is recommended that a morning serum and a late-evening salivary cortisol or 24 hour UFC be measured and shown to be increased prior to proceeding with IPSS. Prior authors have suggested that out-of-cycle IPSS in cyclical disease of other causes than pituitary could cause a false-positive for Cushing disease due to lack of suppression of corticotrophs, meaning that since the corticotrophs are no longer suppressed by high cortisol, after giving $\mathrm{CRH}$ the ACTH and cortisol would increase. ${ }^{2}$ In a review of the value of prolactin measurement in IPSS to document adequate catheterization of the petrosal sinuses, Sharma and colleagues provided a brief description of potentially false-positive IPSS results for CD in a patient later diagnosed as EAS. ${ }^{9}$ The ipsilateral IPS:P prolactin ratio was reported as low suggesting possible inadequate catheterization of the IPS and a repeat IPSS during a cycle of hypercortisolism was not necessary as the patient was subsequently diagnosed by other means as EAS. While a limitation of our report is that prolactin levels were not measured during IPSS and the left IPS was unable to be accessed due to occlusion during the "in-cycle" IPSS study, we were able to demonstrate results of IPSS when performed twice in the same patient, first during a phase of adrenal insufficiency (out-of-cycle) and later during a cycle of hypercortisolemia, and provide evidence that when results of IPSS are interpreted carefully in CCS, a suspected diagnostic pitfall may be avoided. Our patient's out-of-cycle IPSS showed adrenal insufficiency due to profound suppression of the corticotrophs from the in-cycle hypercortisolism, a finding which makes the interpretation of the peak IPS:P ACTH gradient essentially non-diagnostic and should prompt further investigation during a cycle of hypercortisolism.

Very few cases of bronchial typical carcinoid producing ectopic ACTH and causing cyclical Cushing's syndrome have been reported in the literature..$^{10,11}$ To the best of our knowledge this is the first case report demonstrating proof of principle of results of IPSS when performed both in and out-of -cycle in CCS due to EAS, thereby providing a basis for a recommended approach to interpretation of results to prevent misdiagnosis of ectopic ACTH production as Cushing's disease.

\section{Acknowledgements}

We would like to thank Dr. Lynnette Nieman and colleagues at NIH Bethesda, Maryland for their clinical involvement in the case.

\section{Conflicts of interest}

The authors declare that there are no conflicts of interest.

\section{Funding}

A.Y.K has received funding for research from Corcept Therapeutics.

\section{References}

1. Mullan KR, Atkinson AB, Sheridan B. Cyclical Cushing's syndrome: an update. Curr Opin Endocrinol Diabetes Obes. 2007;14(4):317-322.

2. Atkinson B, Mullan KR. What is the best approach to suspected cyclical Cushing syndrome? Strategies for managing Cushing's syndrome with variable laboratory data. Clin Endocrinol (Oxf). 2011;75(1):27-30.

3. Alexandraki KI, Kaltsas GA, Isidori AM, et al. The prevalence and characteristic features of cyclicity and variability in Cushing's disease. Eur J Endocrinol. 2009;160(6):1011-1018.

4. Leal-Cerro A, Martín-Rodríguez JF, Ibáñez-Costa A, et al. Desmopressin test in the diagnosis and follow-up of cyclical Cushing's disease. Endocrinol Nutr. 2014;61(2):69-76.

5. Meinardi JR, Wolffenbuttel BH, Dullaart RP. Cyclic Cushing's syndrome: a clinical challenge. Eur J Endocrinol. 2007;157(3):245-254.

6. Graham UM, Hunter SJ, McDonnell M, et al. A comparison of the use of urinary cortisol to creatinine ratios and nocturnal salivary cortisol in the evaluation of cyclicity in patients with Cushing's syndrome. $J$ Clin Endocrinol Metab. 2013;98(1):E72-E76.

7. Manenschijn L, Koper JW, van den Akker EL, et al. A novel tool in the diagnosis and follow-up of (cyclic) Cushing's syndrome: measurement of long-term cortisol in scalp hair. $J$ Clin Endocrinol Metab. 2012;97(10):E1836-E1843.

8. Lad SP, Patil CG, Laws ER, et al. The role of inferior petrosal sinus sampling in the diagnostic localization of Cushing's disease. Neurosurg Focus. 2017;23(3):E2

9. Sharma ST, Raff H, Nieman LK. Prolactin as a marker of successfu catheterization during IPSS in patients with ACTH-dependent Cushing's syndrome. J Clin Endocrinol Metab. 2011;96(12):3687-3694.

10. Arnaldi G, Mancini T, Kola B, et al. Cyclical Cushing's syndrome in a patient with a bronchial neuroendocrine tumor (typical carcinoid) expressing ghrelin and growth hormone secretagogue receptors. $J$ Clin Endocrinol Metab. 2003;88(12):5834-5840.

11. Cameron CM, Roberts F, Connell J, et al. Diffuse idiopathic pulmonary neuroendocrine cell hyperplasia: an unusual cause of cyclical ectopic adrenocorticotrophic syndrome. Br J Radiol. 2011;84(997):e14-e17. 